\title{
Purely Organic Single Crystal Exhibiting High Hole Mobility for Direct Detection of Ultralow-Dose X-Radiation
}

Dou Zhao ${ }^{1}$, Meng Xu ${ }^{1}$, Bao Xiao ${ }^{1}$, Binbin Zhang ${ }^{1}$, Lei Yan $^{2}$, Guoqiang Zeng ${ }^{2}$, Alain Dubois ${ }^{1,3}$, Paul Sellin ${ }^{4}$, Wanqi Jie ${ }^{1}$, and Yadong $\mathrm{Xu}^{1 *}$

${ }^{1}$ Key Laboratory of Radiation Detection Materials and Devices, Ministry of Industry and Information Technology, School of Materials Science and Engineering \& State Key Laboratory of Solidification Processing, Northwestern Polytechnical University, Xi'an, Shaanxi 710072, China.

${ }^{2}$ Nuclear Technology Key Laboratory of Earth Science, Chengdu University of Technology, Chengdu, 610051, China.

${ }^{3}$ Laboratory of Physical Chemistry - Matter and Radiation, Sorbonne Universite, France.

${ }^{4}$ Department of Physics, University of Surrey, UK.

*Correspondence: xyd220@nwpu.edu.cn,

\begin{abstract}
Precise detection of low-dose X-ray radiation by purely organic direct detectors is vital for tissue-equivalent dosimeters and safety control in medical radiation treatment, but it still remains a challenge. Here, we report on a promising organic radiation detector based on 4-hydroxycyanobenzene $\left(4 \mathrm{HCB}, \mathrm{C}_{7} \mathrm{H}_{5} \mathrm{NO}\right)$ single crystal. Plate-like $4 \mathrm{HCB}$ single crystals up to $18 \times 15 \times 1.2 \mathrm{~mm}^{3}$ in size are obtained by the optimized solvent evaporation method, giving us greater clarification of the two-dimensional nucleation growth mechanism. After post surface treatment, the leakage current of our $4 \mathrm{HCB}$ detectors is no greater than $0.1 \mathrm{pA}$ under an electric field of $600 \mathrm{~V} \mathrm{~cm}^{-1}$. The fabricated detectors show a capability of detecting ${ }^{241} \mathrm{Am} 5.49 \mathrm{MeV} \alpha$ particles with a a well resolved full energy peak. The calculated hole mobility $\left(\mu_{h}\right)$ and hole mobility lifetime product $(\mu \tau)_{h}$ are $3.40 \mathrm{~cm}^{2} \mathrm{~V}^{-1} \mathrm{~s}^{-1}$ and $8.50 \times 10^{-5} \mathrm{~cm}^{2} \mathrm{~V}^{-1}$, respectively. Simultaneously, under a $50 \mathrm{kVp}$ X-ray beam, a detection limit as low as $0.29 \mu \mathrm{Gy}_{\text {air }} \mathrm{s}^{-1}$ with a high sensitivity of $10 \mu \mathrm{C} \mathrm{Gyair}{ }^{-1} \mathrm{~cm}^{-2}$ is achieved at the bias range of 40-100 V, which contributes to a superior X-ray imaging capability with a spatial resolution of $0.9 \mathrm{lp}$ $\mathrm{mm}^{-1}$ at a low-dose rate (below $150 \mu \mathrm{Gy}_{\text {air }} \mathrm{s}^{-1}$ ).
\end{abstract}




\section{Introduction}

$\mathrm{X}$-ray detection is vital for medical diagnosis, scientific research and industrial inspiration $^{1,2}$. The state-of-the-art solid state X-ray semiconductor detectors are, normally, based on the inorganic materials such as silicon ${ }^{3}$, cadmium zinc telluride $(\mathrm{CdZnTe})^{4,5}$, mercury iodide $\left(\mathrm{HgI}_{2}\right)^{6}$ and the emerging metal halide perovskites ${ }^{7-11}$. However, each of these has disadvantages, including inability to cover large curved areas, toxicity, mechanical inflexibility, complex and expensive growth facilities as well as grown-in imperfections induced by high temperature. Additionally, such inorganic materials usually possess a large atomic number and high mass density thus producing a high stopping power for X-rays and lack of tissue-equivalence ${ }^{12-14}$. Tissue equivalence, meaning the effective atomic number $\left(Z_{e f f}\right)$ of the detector material is similar to the average human tissue $Z$ (7.64 for muscles), is particularly important for personal dosimetry, and for dosimetry in radiotherapy and radiobiology ${ }^{15}$. Only when the $Z_{\text {eff }}$ of the dosimeter is matched to the value of human tissue can the dose be obtained without any correction. Furthermore, tissue equivalent detectors with low attenuation efficiency can also be placed between the X-ray source and the patient, allowing a highly localized, real-time radiation exposure monitoring.

Recently, the interest in tissue-equivalent radiation detectors have been boosted by the development of organic semiconductors, such as semiconducting polymers ${ }^{16-19}$ (e.g. poly (triarylamine) (PTAA) thick film), organic semiconducting single crystals $(\mathrm{OSSCs})^{20-23}$ (e.g. 4-hydroxycyanobenzene (4HCB)) and inorganic hybrid materials ${ }^{24,}$ 25 (e.g. polymer blended with Bismuth(III) oxide $\left(\mathrm{Bi}_{2} \mathrm{O}_{3}\right)$ nanoparticles), etc. These devices have been proven to be new options for sensitive and robust X-ray detection materials, possessing great potential as solid-state tissue-equivalent dosimeters operated at room-temperature. They could be used in a vast variety of fields, spanning from radiotherapy and health diagnostics applications, to civil security and industrial application $^{20}$. However, the sensitivity and detection limit are restricted by their low attenuation efficiency and low carrier mobility ${ }^{25}$. Previously reported organic radiation 
detectors are normally operated with a X-ray dose rate of several to tens of $\mathrm{mGy} \mathrm{s}^{-1}$, which is too high to satisfy real applications, e.g., X-ray security screening systems require detectors that are sensitive to a dose limit of $0.25 \mu \mathrm{Gy}^{26}$. Furthermore, use of a high dose rate in medical application is harmful to human tissues. An increased cancer risk associated with X-ray procedures (such as X-ray tomography) has been revealed, especially for children ${ }^{27}$.

In order to increase the sensitivity and reduce the detection limit, nanoparticles, such as bismuth oxide, have been embedded in the organic bulk heterojunction. As a result, the high sensitivity of $1712 \mu \mathrm{C} \mathrm{mGy}{ }^{-1} \mathrm{~cm}^{-3}$ and the latest record of detectable dose rate down to $27 \mu \mathrm{Gy} \mathrm{s}^{-1}$ have been realized ${ }^{25}$. However, such devices are no longer tissue-equivalent due to the presence of high- $\mathrm{Z}$ nanoparticles. Furthermore, the grain boundaries produced by nanoparticles are detrimental for the carrier transport behaviour, typically leading to a longer response time (up to a few seconds) and limited detection performance at dose rates lower than $27 \mu \mathrm{Gy} \mathrm{s}^{-1}$.

In contrast, the purely organic semiconducting single crystals (OSSCs) have been proven to have high carrier mobility as optoelectronic devices due to their well-defined structure, and high purity ${ }^{28,29}$. Among various OSSCs, the 4HCB single crystal semiconductor, possessing a low intrinsic carrier density due to the ultra-wide band gap $\left(E_{g}=4.2 \mathrm{eV}\right)$. Previous reports have recored a relatively low background current $(2.1 \mathrm{nA}$ at bias of $20 \mathrm{~V}$ ) and thus a high signal-to-noise ratio ${ }^{30}$. This material's fast linear response to X-rays and notable radiation hardness and resistance to aging have also been reported ${ }^{20}$. A sensitivity of $0.15 \mu \mathrm{C} \mathrm{Gy}^{-1}$ and a minimum detectable dose rate of $50 \mu \mathrm{Gy} \mathrm{s}^{-1}$ were achieved under a $10 \mathrm{keV}$ synchrotron X-ray beam, indicating the possibility of tissue-equivalent dosimeters with a suitable sensitivity and low detection $\operatorname{limit}^{22}$. However, for the $30 \mathrm{keV} \mathrm{X}$-ray beam that is commonly used for medical therapy and imagery (mammography: 20-30 keV) ${ }^{22}$, the detection performance becomes worse due to lower attenuation efficiency (Fig. S1). Therefore, to achieve higher attenuation efficiency and better X-ray response performance, many improvements of the crystal 
growth, or the device fabrication process, for large-volume and high-quality 4HCB single crystals are needed.

In this work, we report on the development of high-quality and large-volume 4HCB single crystals with unprecedented features for radiation detection. The surface morphology and microscopic defects are investigated, from which the crystal growth mechanism is revealed. Simultaneously, a temperature controlled solvent evaporation method is proposed. Post surface treatment is then carried out and examined by optical transmission spectroscopy and leakage current characterization. Finally, purely organic devices based on the resulting crystals are evaluated as direct detectors of alpha particles and a $50 \mathrm{kVp}$ X-ray beam. Furthermore, these devices show an exceptional stability under long period of continuous operation with superior X-ray imaging capability.

\section{Results and Discussion}

The growth method of 4HCB single crystal is illustrated in Fig. 1a and described in detail in the experimental section. Transparent crystals with sizes up to $18 \times 15 \times 1.2$ $\mathrm{mm}^{3}$ are obtained, as shown in Fig. 1b, with the powder XRD pattern shown in Fig. S2. The preferred growth orientation is determined using $\mathrm{X}$ ray diffraction method (XRD), revealing the dominant $\{002\}$ peaks (Fig. 1c). The calculated distance of a unit-cell along $c$ axis is $25.152 \AA$, which is consistent with the reported value $25.460 \AA^{31}$. The plate-like shape originates from the crystallographic structure: in a unit cell, benzene rings are overlapped along $a$ axis and $b$ axis, while 4HCB molecules are packed by weak hydrogen bonds (between single-molecules) and Van der Waals' force (between bi-molecular chains) along $c$ axis $^{30,32}$ (Fig. 1d), in which $a=9.190 \AA, b=10.750 \AA, c$ $=25.460 \AA$ A . According to the Periodic Bond Chain $(\mathrm{PBC})$ theory ${ }^{33}$, the relaxation time for absorption the growth unit is determined by the bond energy, which in turn is associated with the growth rate and the specific shape. DSC analysis has been done to evaluate the thermo-stability, illustrating the high thermo-stability under normal device 
operation (at room temperature).
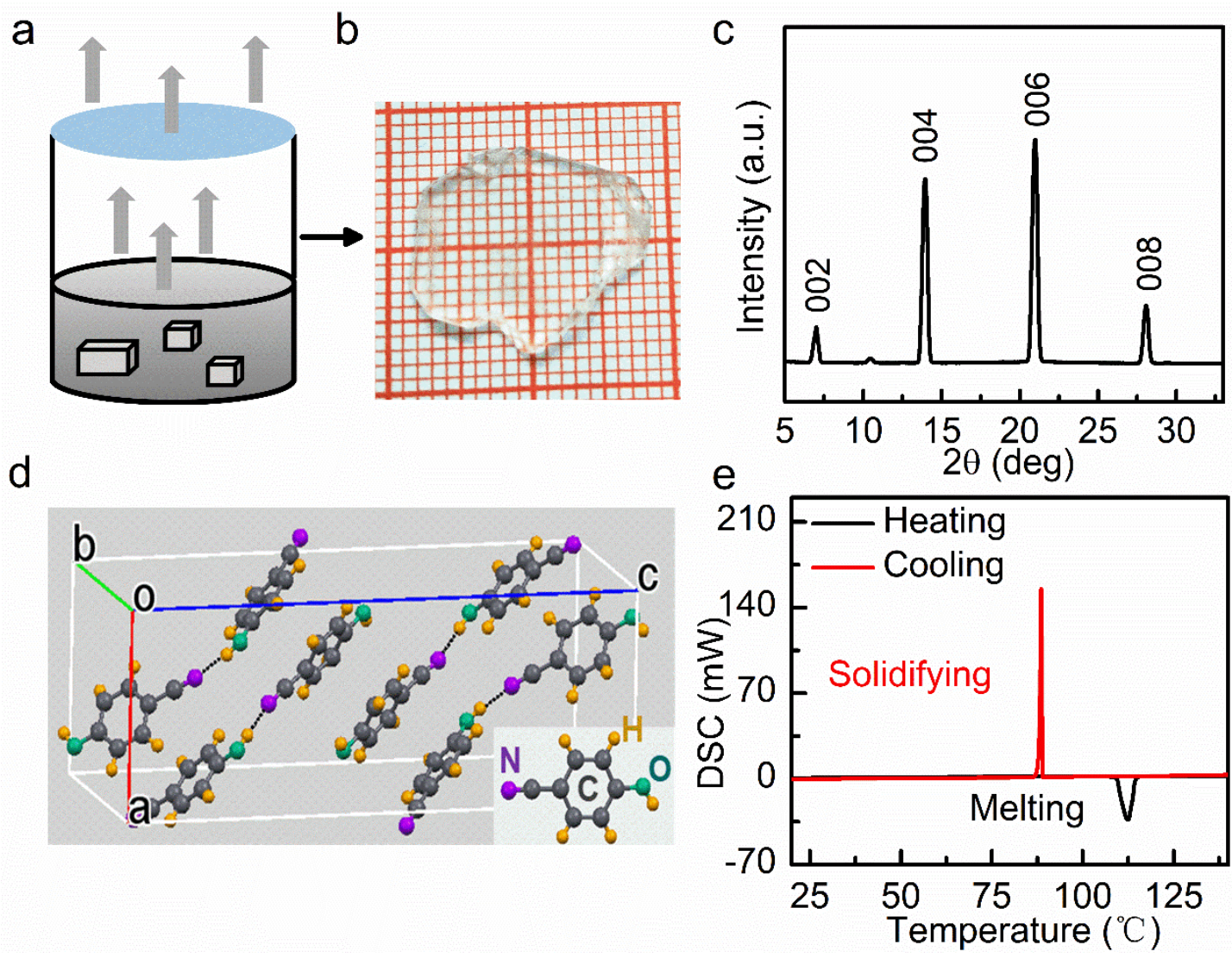

Figure 1. 4HCB single crystal growth and characterization (a) The growth schematic,

(b) Photograph of the as-grown crystal (transparent), (c)Single crystal XRD pattern, (d) $4 \mathrm{HCB}$ crystallographic structure along $c$ axis (with deleting some un-bonded 4HCB molecules in a unit cell for clarity), (e) DSC curve of heating and cooling process.

The morphological characterizations are carried out using an optical microscope and an Atomic Force Microscope (AFM). The freely grown surface is shown in Fig. 2a, whilst the crystal surface that is in contact with the bottom of the beaker is shown in Fig. 2b. Extensive irregular cracks are observed on the surface in contact with the beaker. The typical micrographs of freely grown surface are characterized by three regions marked by I, II, III. Region I is a mirror-like smooth surface, in contrast, region III is full of macro layered structures. Region II is smooth in general excepting for a few shallow pits (Fig. 2a). The tapping mode of the AFM is used to scan the $10 \mu \mathrm{m} \times 10$ $\mu \mathrm{m}$ area of the central flat surface in Fig. 2a. Two typical step-like structures are observed, as shown in Fig. 2c and 2d with the corresponding cross-section curves, 
respectively. In Fig. 2c, there are 5 steps and a slope (C) within the planar dimension of $10 \mu \mathrm{m} \times 10 \mu \mathrm{m}$, and the height of steps are $2.5 \mathrm{~nm}$ (step A), $1.3 \mathrm{~nm}$ (step B). By contrast, there are only steps of $2.5 \mathrm{~nm}$ within the same planar region in Fig. 2d. According to the crystal structure, $d_{(001)}=2.5 \mathrm{~nm}$, the step A and step B are corresponding to unitcell layers and half unit-cell layers along $c$ axis, respectively.
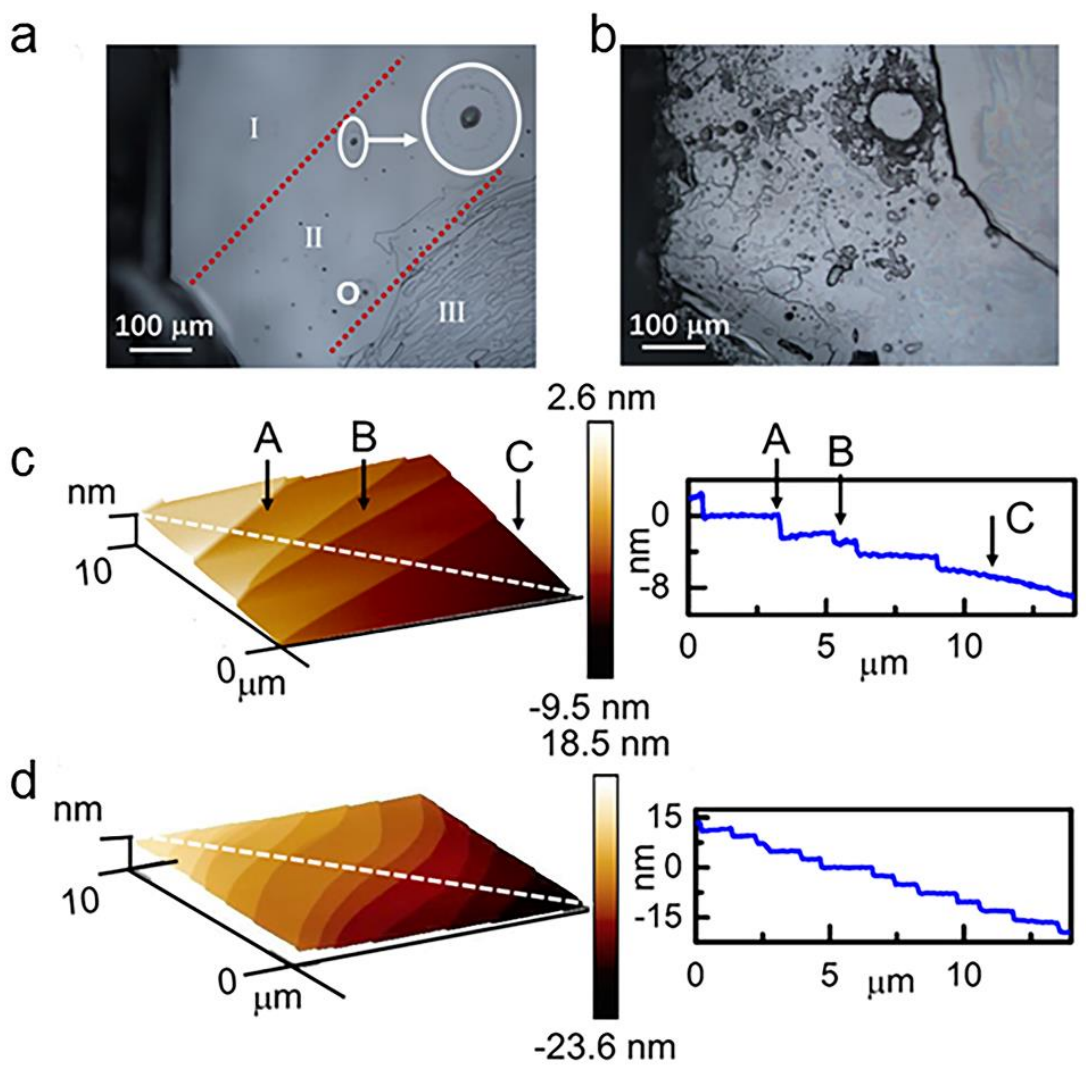

Figure 2. Characterizations of as-grown single crystal by Optical Microscope (a) The free surface growth, (b) The surface initiated by the beaker bottom, AFM tapping picture of 4HCB surface and corresponding cross-section curve (c), (d) with slow and large growth rate of [001] direction, respectively.

Previous work on various organic single crystals, such as pentacene, 2,5-bis(4biphenylyl)thiophene (BP1T), etc ${ }^{34-36}$, illustrate that these micro- and macro- step-like structures in Fig. 2 originated from the two-dimensional (2D) nucleation growth mechanism. In our experiment, the evaporation rate of ethylic ether (EE) is high. A high degree of supersaturation can be easily achieved, which is essential for $2 \mathrm{D}$ nucleation mechanism growth, therefore, this mechanism dominates the growth process here. 
In terms of this growth pattern, we note that the growth rate of [001] direction in Fig. $2 d$ is larger than that in Fig. 2c, which is attributed to a higher supersaturation. In Fig. 2d, the four-molecular chains (length: $2.5 \mathrm{~nm}$ ) as the growth units is indicated by the step-like structure of $2.5 \mathrm{~nm}$. Similarly, the growth unit of bi-molecular chains (length: $1.25 \mathrm{~nm}$ ) can be deduced from Fig. 2c. The abundance of these two units in the solution is controlled by the supersaturation. In the lower-supersaturation solution, the bi-molecular chains and four-molecular chains could be formed as the basic growth units. However, in the higher supersaturation solution, the concentration of fourmolecular chains is higher, which is the dominant basic growth units. Therefore, a growth model for 4HCB single crystal is proposed, as shown in Fig. 3a. With increasing supersaturation $(\beta)$, at first, single-molecules are connected by hydrogen bonds and bimolecular chains are generated, then the four-molecular chains are composed by Van der Waals' force considering assembly bi-molecular chains. Finally, once the $\beta$ reaches to $\beta^{*}$ (critical supersaturation), the $2 \mathrm{D}$ nucleus is formed and the $4 \mathrm{HCB}$ single crystal start to growth layer by layer along the [001] direction.

In case of the $2 \mathrm{D}$ nucleation growth, the growth control method had been discussed $^{37,38}$. The growth rate $(R)$ of $(001)$ face of $4 \mathrm{HCB}$ for mononuclear mechanism is given as,

$$
R=k(T) \beta^{\frac{1}{2}} \exp \left(-\frac{\Delta G}{k_{B} T}\right)
$$

where $k(T)$ is a kinetic constant depending on the temperature and supersaturation ratio $\beta=C / C s$ ( $C$ is the concentration in solution and $C s$ is the solubility), $\Delta G$ is the Gibbs energy for growth and $k_{B}$ is the Boltzmann constant.

In general, $\Delta G=\frac{4 \lambda^{2}}{k_{B} T \ln \beta}$ ( $\lambda$ is the edge free energy (per molecule) of the nucleus), when only considering the change of $R$ with $\beta$, then:

$$
R=\mu(T) \exp \left(-\frac{b}{\ln \beta}\right)
$$

The change of $R$ with $\beta$ is mainly revealed in $\exp \left(-\frac{b}{\ln \beta}\right)$, while $\mu(T)$ reveals the influence of the temperature, as shown in Fig. $3 b$. 
An incubation period exists before the relatively high critical supersaturation $\left(\beta^{*}\right)$ condition is reached. Once $\beta$ reaches the critical value $\beta^{*}$, the growth rate increases rapidly. In order to tailor the nucleation and crystallization rate, the kinetic conditions should be considered. Here, the solvent evaporation process and mass transport process during growth are controlled by adjusting the temperature. The preferred growth temperature (from -5 to $10{ }^{\circ} \mathrm{C}$ ) is explored. Two typical results are shown in Fig. $3 \mathrm{c}$. The planar size of crystals grown at $5{ }^{\circ} \mathrm{C}$ (I) is $4-6 \mathrm{~mm}$ and those at $-5{ }^{\circ} \mathrm{C}$ (II) is larger than $10 \mathrm{~mm}$. Due to the slower growth rate at $-5^{\circ} \mathrm{C}$, the obtained crystals have a higher crystal quality with fewer macro layered defects and very few defects on the surface (Fig. S3).

a

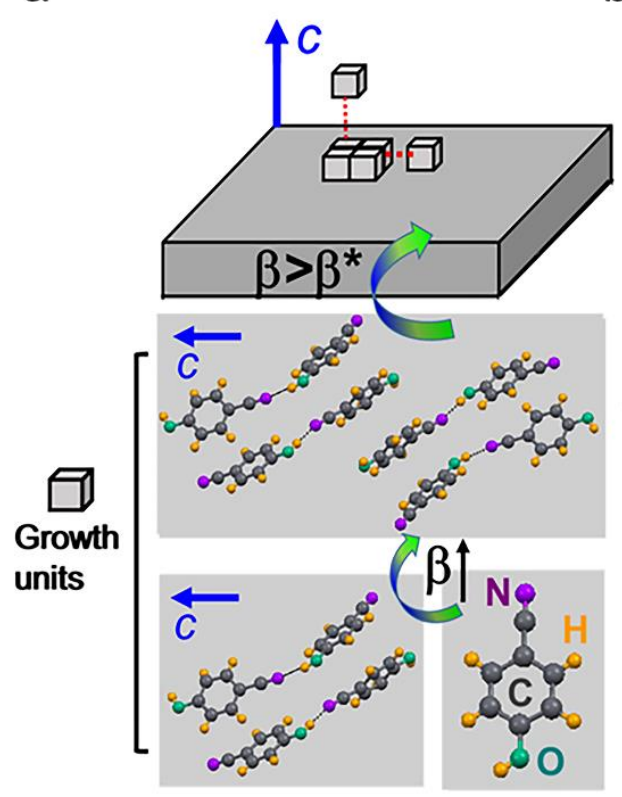

b
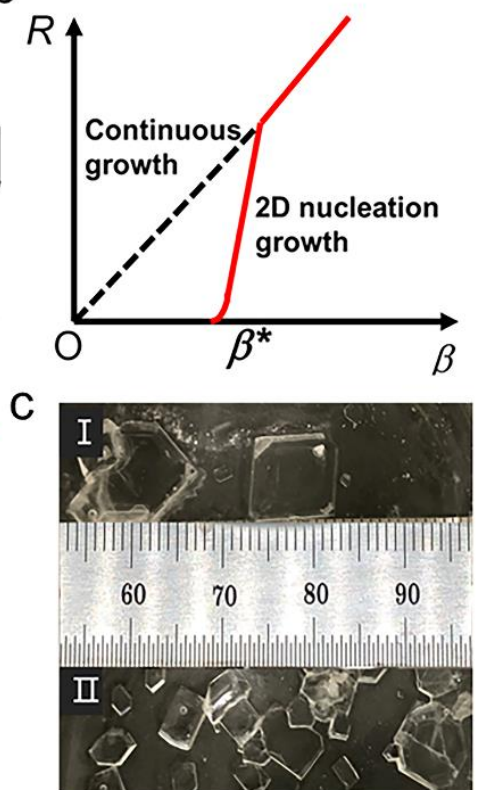

Figure 3. (a) The schematic of $4 \mathrm{HCB}$ crystals growth mechanism; (b) The growth rate $(R) v s$. supersaturation ratio $(\beta)$ (continuous growth (blue dot line) and 2D nucleation growth (red line), (c) Transparent crystals (with a black background) left in a beaker after a growth run ((I) is at $-5^{\circ} \mathrm{C}$ and (II) is at $\left.5{ }^{\circ} \mathrm{C}\right)$.

In addition, the grown-in defects on the surface of $4 \mathrm{HCB}$ crystals have to be taken into account, which critically deteriorate the device leakage current. Therefore, the surface treatment is adopted by subsequently chemo-mechanically polishing, etching 
and passivation prior to performing the measurements. The post treated surface without macro defects is shown in the insertion of Fig. 4a. After the surface treatment, the average UV-Vis transmittance is increased from $75 \%$ to $90 \%$ (Fig. 4 a) in the wavelength range of $200-1000 \mathrm{~nm}$. The band gap $E g$ is calculated as $4.2 \mathrm{eV}$.

The device based on as-grown $4 \mathrm{HCB}$ single crystal materials are fabricated by carbon, silver paste, and the sputtered Au electrodes, with the configuration is shown in Fig. $4 \mathrm{~b}$. The $J-E$ curves for these three types of electrodes are close, given a resistivity on the order of $10^{12} \Omega \cdot \mathrm{cm}$, seen in Fig. 4c. To avoid the emission of secondary electrons from high-Z electrodes, the carbon electrode is employed to evaluate the intrinsic $\mathrm{X}$ ray detection property of $4 \mathrm{HCB}$ single crystal. The $J-E$ curves for both as-grown and post surface treated crystals are compared in Fig. $4 \mathrm{~d}$. After the surface treatment, the surface leakage current density decreased to $10^{-10} \mathrm{~A} \mathrm{~cm}^{-2}$ at an electric field of $10^{4} \mathrm{~V}$ $\mathrm{cm}^{-1}$ (Fig. S4), which is 100 times lower than that for as-grown crystals $\left(10^{-8} \mathrm{~A} \mathrm{~cm}^{-2}\right)$.

a
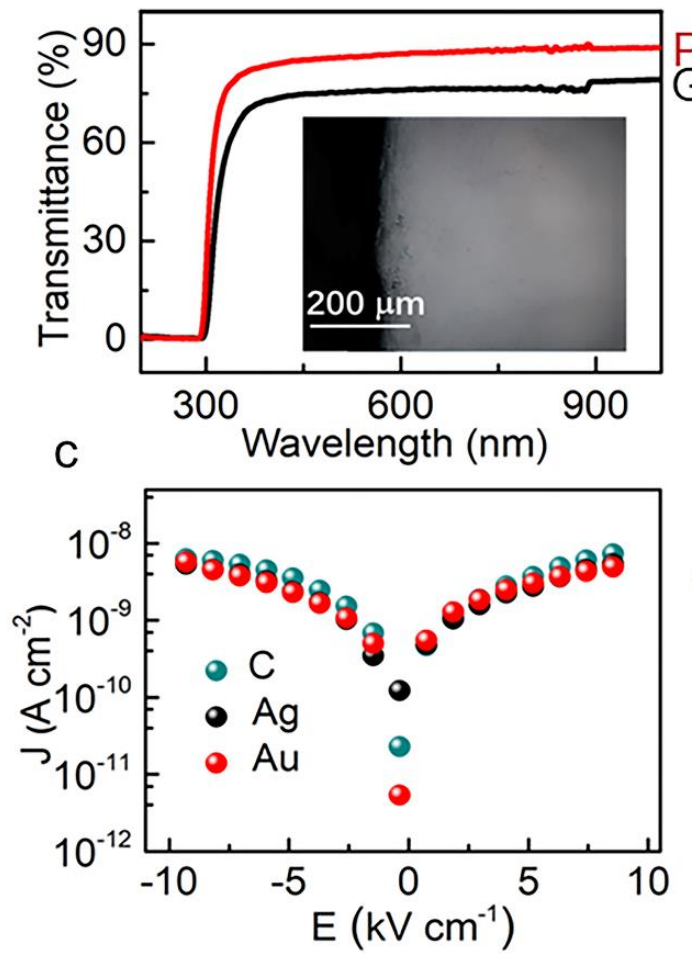

b
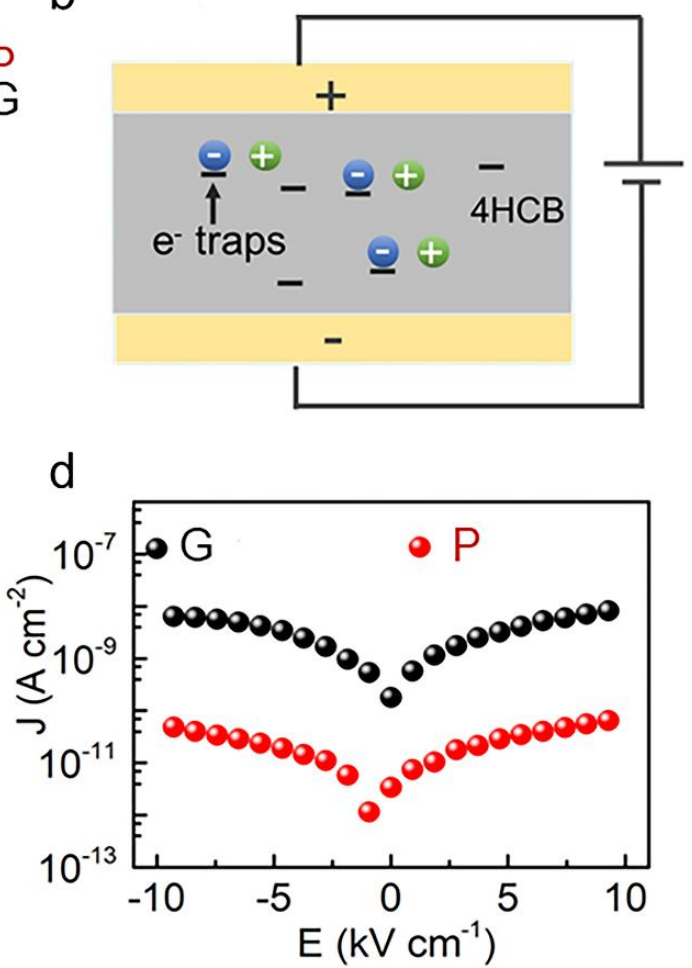

Figure 4. (a) UV-Vis spectra of as-grown $(\mathrm{G})$ and post surface treatment $(\mathrm{P})$ crystals (insertion is the surface characterizations of polished crystal by Optical Microscope), (b) Sandwich electrodes configuration of device, (c) Current density (J) vs. Electrical 
field $(E)$ curves of as-grown crystals with different electrodes, (d) $J-E$ curves of asgrown $(\mathrm{G})$ and post surface treatment $(\mathrm{P})$ crystals with Carbon electrodes.

The schematic of alpha particles detection is shown in Fig. 5a. The mean energy loss $(35.9 \mu \mathrm{m})$ in air is calculated by the software package SRIM ${ }^{39}$ (Fig. S5). Fig. 5b shows the pulses in $4 \mathrm{HCB}$ OSSCs devices induced by the ${ }^{241} \mathrm{Am}$ alpha particles (A $\left.=2.32 \times 10^{5} \mathrm{~Bq}\right)$ incident from the anode. The pulse fall time for hole signals $\left(t_{f}, 10 \%\right.$ $90 \%$ of the full amplitude) has been measured, then the histogram is plotted using 3000 pulses, the typical results under $600 \mathrm{~V}$ bias is shown in Fig. 5c. The results under other biases are shown in Fig. S6. For photo-generated carriers drifting across the thickness, the mobility $\mu$ is given by:

$$
\mu=\frac{v_{d r}}{E}=\frac{d^{2}}{V \times t_{f}}
$$

where $v_{d r}$ is the drift velocity, $E$ is the electric field, $d$ is the thickness of the crystals, $V$ is the bias voltage and $t_{f}$ is the drift time. The hole mobility along the $c$ axis is estimated as $\sim 3.4 \mathrm{~cm}^{2} \mathrm{~V}^{-1} \mathrm{~s}^{-1}$, which is the highest value among organic detectors based on the alpha particles characterization ${ }^{40}$, as shown in Fig. 5 d.

More importantly, the full-energy peaks are resolved over the bias voltage of 200$600 \mathrm{~V}$ at room temperature, as seen in Fig. 5e. An energy resolution of $45 \%$ (full width at half maximum (FWHM)) is achieved at $600 \mathrm{~V}$ bias. The position of the peak centroid shifts toward higher channels with increasing bias voltage, revealing the efficient charge collection. The position shifts of the Gaussian peak centroid extracted from the pulse height spectra is plotted in Fig. 5f, as a function of bias voltage. The peak centroid channel is proportional to the charge collection efficiency $\eta$ (i.e., the fraction of energy detected over the total theoretical energy release in the material). The centroid peak tends to saturate at high voltages, with a well-known behavior following the Hecht plot function:

$$
\eta=\frac{\mu \tau V}{d^{2}}\left(1-\exp \left(-\frac{d^{2}}{\mu \tau V}\right)\right)
$$

where $V$ is the applied voltage, $d$ is the thickness of detector, and $\mu \tau$ is the mobilitylifetime product of the detector material. The fitting of the experimental data is reported 
by the red lines in Fig. $5 \mathrm{f}$, with the $(\mu \tau)_{h}$ of $8.50 \times 10^{-5} \mathrm{~cm}^{2} \mathrm{~V}^{-1}$, which is a much higher value than $1.90 \times 10^{-6} \mathrm{~cm}^{2} \mathrm{~V}^{-1}$ reported in reference ${ }^{40}$.

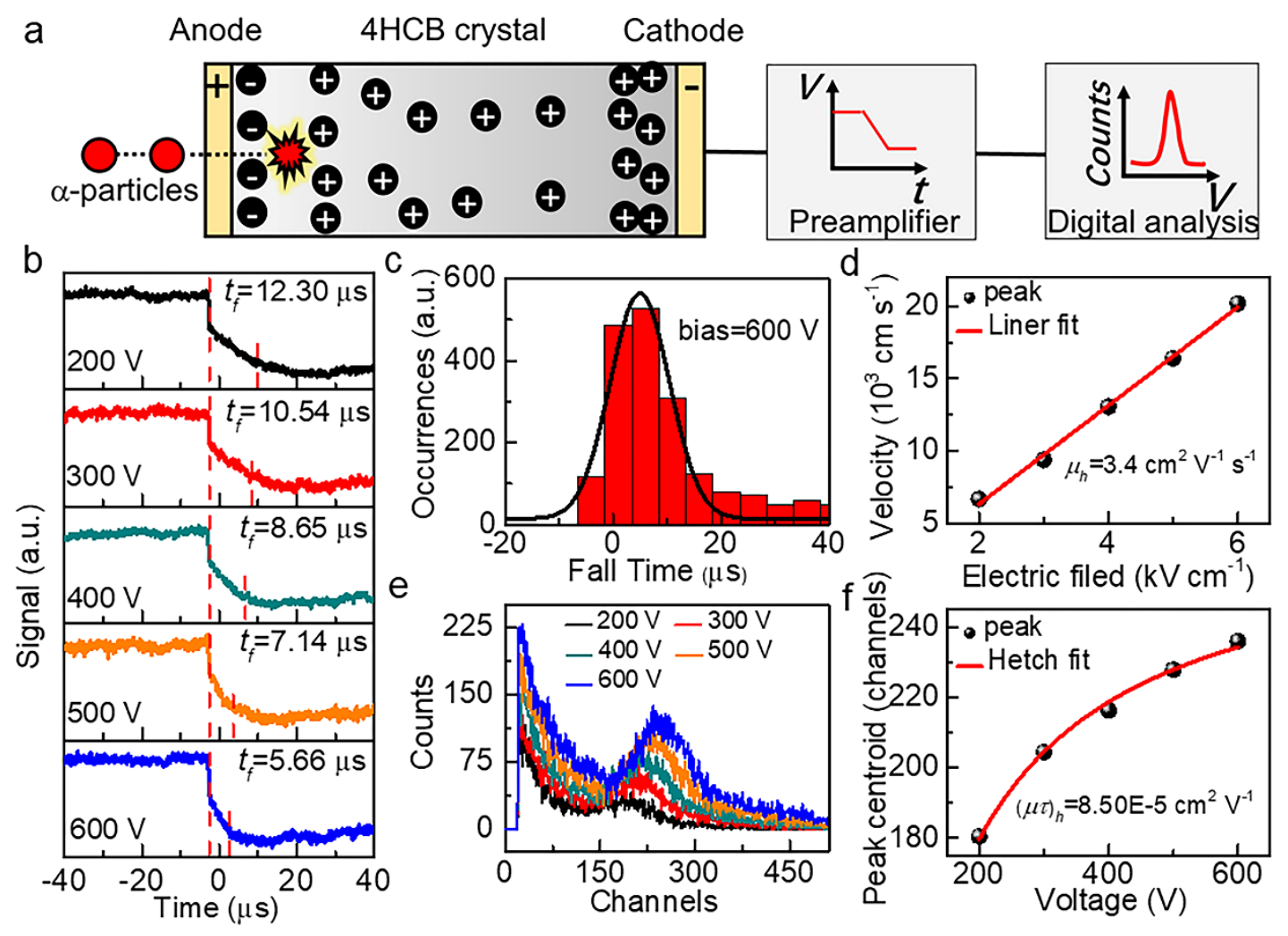

Figure 5. (a) The schematic of alpha particles detection, (b) ${ }^{241} \mathrm{Am}$ alpha pulses in 4HCB single crystal at a series of bias voltage (vertical red lines indicate the fall time $\left.\left(t_{f}\right)\right)$, (c) Pulse fall time histogram of 4HCB single crystal biased at $600 \mathrm{~V}$, (d) Mobility value extrapolation from drift velocity vs. electric field linear plot, (e) Pulse height spectra obtained with digital pulse shape analysis at various bias voltage, (f) Gaussian peak centroid position vs. applied voltage plot (blue dot) and Hecht equation fitting.

For the normal medical imaging application, the performance under $50 \mathrm{kVp} \mathrm{X}$-ray beam is important, mainly including sensitivity and detection limit. Generally, the sensitivity is calculated as definition,

$$
S=\frac{\Delta J}{D}, \Delta J=\frac{I_{O N}-I_{O F F}}{A}
$$

where $I_{O N}$ and $I_{O F F}$ is the current measured under and without the X-radiation respectively, $A$ is the area of electrodes, and $D$ is the incident X-radiation dose rate in air. The detection limit is defined as the dose rate that the signal-to-noise rate (SNR) 
value of the signal current is larger than 3 defined by the IUPAC ${ }^{41}$, details in Supplementary Discussion 1.

Fig. 6a shows the typical Photocurrent-Time (I-T) curve of 4HCB device based on the post-surface treated single crystals recorded in a dose rate range of $0.29-28.8 \mu \mathrm{Gy}$ air $\mathrm{s}^{-1}$ under an electrical field strength of $600 \mathrm{~V} \mathrm{~cm}^{-1}$. No hysteresis or appreciable current drift is observed, which is comparable with other highly performed detectors ${ }^{42-45}$. Similar $I-T$ curves are obtained at electric field of 400,800 and $1000 \mathrm{~V} \mathrm{~cm}^{-1}$ (Fig. S8). The dark current is stable after repeated exposure under X-ray beam, with a value of $0.1 \mathrm{pA}$ at $600 \mathrm{~V} \mathrm{~cm}^{-1}$. From the $I-T$ curves, the linear Photocurrent density - Dose rate (0.29-8.6 $\left.\mu \mathrm{Gyair}^{-1}\right)(\Delta J-D)$ relation is revealed (Fig. 6b). The sensitivity in the range of $0.29-8.6 \mu \mathrm{Gy}_{\text {air }} \mathrm{S}^{-1}$ is calculated by the slope of $\Delta J-D$ curve (Fig. S9). At an electric field of $1000 \mathrm{~V} \mathrm{~cm}^{-1}$, a sensitivity of $10 \mu \mathrm{C} \mathrm{Gyair}^{-1} \mathrm{~cm}^{-2}$ (Fig. 6b) is realized. It is noteworthy that the stable and repeatable detectable dose rate $0.29 \mu \mathrm{Gyair}^{-1}$ has been achieved (Fig. 6a insertion). In order to assess the detection limit, the SNR values of the signal current (dose rate, $0.29 \mu \mathrm{Gy}_{\text {air }} \mathrm{s}^{-1}$ ) are calculated, as shown in Fig. 6c, which are between 9 and 24 , larger than the defined value of 3 . Therefore, one can deduce that the minimum detectable dose rate is lower than $0.29 \mu \mathrm{Gy}_{\text {air }} \mathrm{s}^{-1}$, which is one magnitude lower than that of the device based on as-grown crystals, more details in Fig. S7. This value is even smaller than the reported $0.5 \mu \mathrm{Gy}_{\text {air }} \mathrm{s}^{-1}$ in $\mathrm{MAPbBr}_{3}$ single crystals ${ }^{8}$ and about 19 times lower than what required for regular medical diagnostics $\left(5.5 \mu \mathrm{Gy}_{\text {air }} \mathrm{s}^{-1}\right)^{46}$.

Both the low dark current noise and highly stable SNR results in a very low level of baseline drift and highly stable photocurrent output signal, therefore, the 4HCB detector exhibit superior imaging capability. As shown in Fig. 6e, the X-ray image of a metallic nut (Fig. 6d) is recorded at an electric field of $100 \mathrm{~V} \mathrm{~cm}^{-1}$ with the exposure dose rate of $50 \mu \mathrm{Gyair}^{-1}$. The high colour contrast demonstrates that the object is clearly resolved, furthermore, it shows unambiguously the similarity in shape and size between the nut and the X-ray image which means that there is no variation or deviation. Finally, the spatial resolution of $0.9 \mathrm{lp} \mathrm{mm}^{-1}$ is achieved based on such device by imaging the 
sharp edge of the line pair card at room temperature, as shown in Fig. S10.
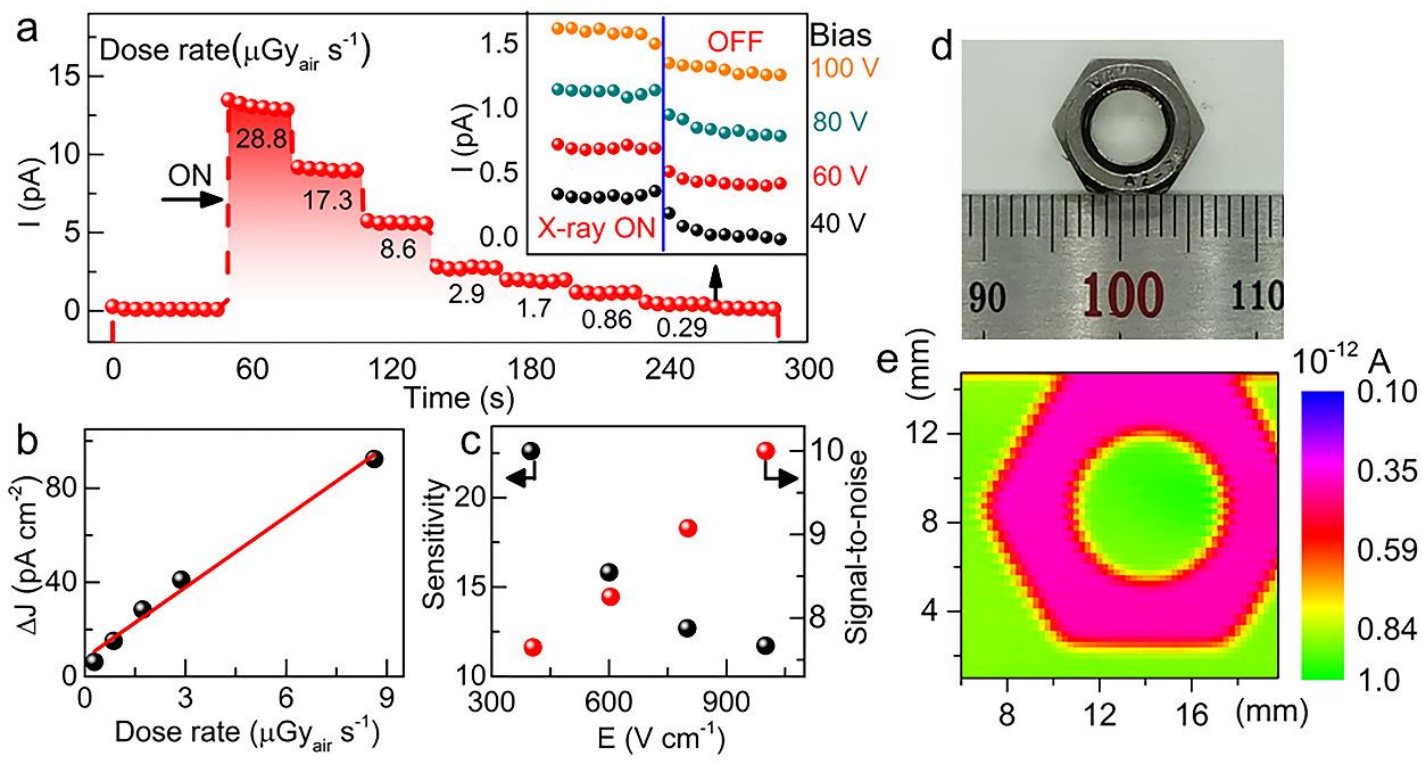

Figure 6. (a) Photocurrent-Time $(I-T)$ curve with changing Dose rate $(D)$ at Electrical field $(E)$ of $0.6 \mathrm{kV} \mathrm{cm}^{-1}$, insertion is the $I-T$ curves (with X-ray beam of $0.29 \mu \mathrm{Gy}_{\text {air }} \mathrm{s}^{-1}$ ) under different bias voltages, (b) $\Delta J$ vs. $D$ (with blue dots) and linear fitting curve (red dashed line), (c) Signal-to-noise (D: $\left.0.29 \mu \mathrm{Gyair} \mathrm{s}^{-1}\right)$ and sensitivity $\left(\mu \mathrm{C} \mathrm{Gyair}^{-1} \mathrm{~cm}^{-2}\right)$ of devices, (d), (e) Photograph and corresponding X-ray image of a metallic nut by the 4HCB detector.

Compared with other detectors $8,21,22,25$, due to low attenuation of the impinging X-ray beam ${ }^{7}$ (Fig. 7a) and low detection limit (Fig. 7b) of 4HCB detector, it can be placed between the X-ray source and the patient, allowing a highly localized, real-time radiation exposure monitoring. This appealing application can be achieved only by the detectors possessing both low detection limit and low stopping power.
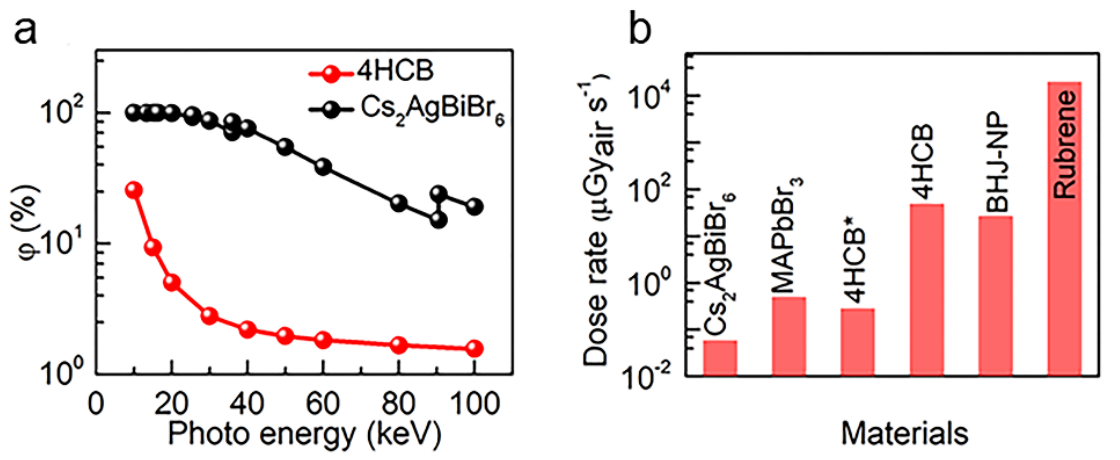

Figure 7. (a) Attenuation efficiency $(\varphi \%)$, (b) Minimum detectable dose rate 
comparison of various materials, $4 \mathrm{HCB}^{*}$ represented the result in this work.

\section{Conclusion}

In conclusion, the two-dimensional nucleation mechanism of purely organic 4HCB crystal has been revealed, from which the centimetre-sized (up to $18 \times 15 \times 1.2$ $\mathrm{mm}^{3}$ ) single crystals were obtained by tailoring the growth temperature. A leakage current of $0.1 \mathrm{pA}$ at $600 \mathrm{~V} \mathrm{~cm}^{-1}$ is achieved after the surface treatment. The fabricated detectors are capable of detecting ${ }^{241} \mathrm{Am} 5.49 \mathrm{MeV} \alpha$ particles with well a resolved full energy peak. The hole mobility and Hetch equation fitted mobility lifetime are $3.4 \mathrm{~cm}^{2}$ $\mathrm{V}^{-1} \mathrm{~s}^{-1}$ and $8.50 \times 10^{-5} \mathrm{~cm}^{2} \mathrm{~V}^{-1}$, respectively. The detection limit as low as $0.29 \mu \mathrm{Gy}$ air $\mathrm{s}^{-}$

${ }^{1}$ with a sensitivity of $10 \mu \mathrm{C} \mathrm{Gyair}{ }^{-1} \mathrm{~cm}^{-2}$ is achieved. In addition, it exhibits superior Xray imaging capability with a spatial resolution of $0.91 \mathrm{p} \mathrm{mm} \mathrm{mm}^{-1}$ thanks to its low dark current, negligible baseline drift, and extremely high stability. The superior X-ray and alpha particles responses and low-cost, large-sized growth method of 4HCB single crystals points to organic single crystals as a promising next-generation material in tissue-equivalent dosimeter and X-ray imaging.

Overall, our work demonstrates how all-organic semiconductors, if carefully engineered, can possess good detection performances that compare to those of inorganic semiconductors. These results and insights will encourage future extensive explore for growth optimization, diverse electronic applications and photoconductive mechanism of organic materials.

\section{Methods}

\section{HCB single crystal growth}

4-hydroxycyanobenzene (4HCB, 98\%) was purchased from Aladdin Reagent Ltd. Ethylic ether (min.99.5\%) was purchased from Sinopharm Chemical Reagent Co., Ltd. and petroleum ether (bp $40-60{ }^{\circ} \mathrm{C}$, min.75\%) was purchased from Kemiou Chemical Reagent Co., Ltd. Both solvents were used without further purification. The raw 4HCB powder was purified by dissolving $4 \mathrm{HCB}$ in ethylic ether (EE) and letting the solvent slowly evaporate. This step left a number of 4HCB crystals, removed to a baker and 
washed with warm petroleum ether (PE). All of the purification steps were repeated at least three times to improve the purity. $4 \mathrm{HCB}$ single crystals growth was carried out as previously reported ${ }^{47}$. Through growth parameters optimization, including solvent ratio (EE:PE=20:1, vol.\%), concentration $(8 \mathrm{mg} / \mathrm{ml})$ and growth temperature $\left(-5-10{ }^{\circ} \mathrm{C}\right)$. Before the solvent entirely flown away, the crystals were removed from the baker almost without any macro damage. In particularly, all of the steps were carried out in ultra-clean chamber to prevent any dust pollution.

\section{Surface Treatment}

The surface treatment is adopted to remove the defects. First, as-grown crystals are polished with $\mathrm{MgO}$ and $\mathrm{SiO}_{2}$ suspension $\left(\mathrm{SiO}_{2}: \mathrm{H}_{2} \mathrm{O}=1: 1\right.$, vol.\%), then etched with a mixed solution consist of a volume mixture of $30 \% \mathrm{EE}$ and $70 \% \mathrm{PE}$ for $1 \mathrm{~min}$. The etching stage produces fresh dangling hydrogen bonds, which are passivated by a treatment with a $\mathrm{H}_{2} \mathrm{O}_{2}(30$ vol.\%) solution for $60 \mathrm{~s}$.

\section{Basic Characterization}

Powder X-ray diffraction (XRD) measurements were performed by grinding one piece of as-grown 4HCB single crystal into fine powder in a mortar. The MAXima_X XRD7000 equipment mainly with $\mathrm{Cu} \mathrm{K} \alpha 1$ was used, scanning angle in range of $5^{\circ}-90^{\circ}$ and scanning rate of $5 \% \mathrm{~min}$. The XRD pattern for $4 \mathrm{HCB}$ single crystal was also measured. The thermal behavior of the as-grown single crystal was evaluated by the MettlerToledo differential scanning calorimeter, over the range of $20{ }^{\circ} \mathrm{C}$ and $140{ }^{\circ} \mathrm{C}$ at the heating rate of $5{ }^{\circ} \mathrm{C} / \mathrm{min}$ in the nitrogen atmosphere and then a controlled cooling of the same condition following. Light micrographs were got with a Nikon LV-100ND microscope. AFM measurements were performed by the Bruker Dimension Icon equipment using SCANASYST-AIR tips (Nano sensors) in non-contact mode. I-V measurements were performed using a Keithley 487 Picoammeter and stabilized bias supply. The sandwiched electrode configuration was fabricated, in which the sample was mounted between two round electrodes of $3 \mathrm{~mm}$ diameters. The different electrode materials were used, including Conductive Silver Paint (SPI-05001-AB), Carbon 
Double-Sided Tape (FN731-8, Nisshin EM Co., Ltd. Resistivity $<5$ ohms $/ \mathrm{mm}^{2}$ ), Copper Double-Sided Tape (FS05085, Resistivity $<0.01 \mathrm{ohms} /$ inch $^{2}$ ). The J-V curve of polished crystal was calculated using Leakage Current-Time (I-T) curve at various bias voltages.

\section{Alpha particles detection by 4 HCB detectors}

The detectors were fabricated with $1 \mathrm{~mm}$ thick 4HCB single crystals with $100 \mathrm{~nm}$ thick sputtered gold electrodes on both sides of crystals and electrodes area of $7 \mathrm{~mm}^{2}$. Irradiation was performed in air at room temperature using a ${ }^{241} \mathrm{Am}$ alpha source, with the particles impinging on the top surface. Bias voltages in the range of $200-600 \mathrm{~V}$ were used in sandwich configuration. The detectors were positioned inside a metal chamber at a distance of $5 \mathrm{~mm}$ from the device. The OSSCs detectors was connected to an ultralow noise charge-sensitive preamplifier ( $\mathrm{eV}$ model 550), with a charge sensitivity of $3.6 \mathrm{mV} / \mathrm{fC}$. Bias voltage was applied via the preamplifier by means of an Ortec 710 power supply. The preamplifier output was directly connected to a digital pulse shaper with long shaping times, in the order of tens of microseconds. A shaping time of $15 \mu \mathrm{s}$ for the CR-RC shaper was used in this work.

\section{X-ray beam detection by $4 \mathrm{HCB}$ detectors}

4HCB single crystals, the planer dimension of $5 \times 5 \mathrm{~mm}^{2}$ crystals with thickness of $1.0 \pm 0.1 \mathrm{~mm}$, were fabricated with Carbon electrodes in sandwich configuration as selfstanding devices. A tungsten target X-ray tube (SPELLMAN XRB011) was used as the source. The X-ray source was operated with tube voltage of $50 \mathrm{kVp}$ (X-ray photo energy up to $50 \mathrm{keV}$ and peak intensity at $22 \mathrm{keV}$ ) and the dose rates of 0.29-28.8 $\mu \mathrm{Gyair} \mathrm{s}^{-1}$. During the experiment, the dose rate of X-ray beam was modulated by changing the current $(0.001-0.6 \mathrm{~mA})$ of X-ray tube, as well as by controlling the distance $(49 \mathrm{~cm})$ between the device and the X-ray source. The X-ray dose rate was carefully calibrated by using both the Geiger counter and the thermos luminescence dosimetry. The bias voltage applied on the device and the induced photocurrent was recorded by a Keithley $6517 \mathrm{~B}$. All the reported measurements were made in air condition at room temperature. 


\section{Acknowledgements}

This work is supported by the National Natural Science Foundations of China (Nos. 51872228,51802262 and U1631116). Project is also supported by the National Key Research and Development Program of China (2016YFE0115200), the Natural Science

Basic Research Plan in Shaanxi Province of China (2019ZDLGY04-07), and the Fundamental Research Funds for the Central Universities (3102017zy057 and 3102018jcc036).

\section{Reference}

1. S. Miyajima, Medical physics, 2003, 30, 771-777.

2. W. Wei, Y. Zhang, Q. Xu, H. Wei, Y. Fang, Q. Wang, Y. Deng, T. Li, A. Gruverman and L. Cao, Nat. Photon., 2017, 11, 315.

3. M. Yaffe and J. Rowlands, Physics in Medicine \& Biology, 1997, 42, 1.

4. Y. Eisen and A. Shor, Journal of crystal growth, 1998, 184, 1302-1312.

5. Y. Eisen, A. Shor and I. Mardor, Nucl Instrum Meth A, 1999, 428, 158-170.

6. S. Swierkowski, G. Armantrout and R. Wichner, Applied Physics Letters, 1973, 23, 281-282.

7. W. Pan, H. Wu, J. Luo, Z. Deng, C. Ge, C. Chen, X. Jiang, W.-J. Yin, G. Niu and L. Zhu, Nat. Photon., 2017, 11, 726.

8. H. Wei, Y. Fang, P. Mulligan, W. Chuirazzi, H.-H. Fang, C. Wang, B. R. Ecker, Y. Gao, M. A. Loi and L. Cao, Nat. Photon., 2016, 10, 333.

9. Y. Xu, B. Jiao, T.-B. Song, C. C. Stoumpos, Y. He, I. Hadar, W. Lin, W. Jie and M. G. Kanatzidis, Acs Photonics, 2018, 6, 196-203.

10. L. Li, X. Liu, H. Zhang, B. Zhang, W. Jie, P. J. Sellin, C. Hu, G. Zeng and Y. $\mathrm{Xu}$, ACS applied materials \& interfaces, 2019, 11, 7522-7528.

11. Y. He, L. Matei, H. J. Jung, K. M. McCall, M. Chen, C. C. Stoumpos, Z. Liu, J. A. Peters, D. Y. Chung, B. W. Wessels, M. R. Wasielewski, V. P. Dravid, A. Burger and M. G. Kanatzidis, Nature Communications, 2018, 9, 1609.

12. P. Cheuvart, U. El-Hanani, D. Schneider and R. Triboulet, Journal of crystal growth, 1990, 101, 270-274.

13. U. Roy, A. Burger and R. James, Journal of Crystal Growth, 2013, 379, 57-62.

14. M. Schieber, M. Roth and W. Schnepple, Journal of Crystal Growth, 1983, 65, 353-364.

15. C. Furetta, M. Prokic, R. Salamon, V. Prokic and G. Kitis, Nuclear Instruments and Methods in Physics Research Section A: Accelerators, Spectrometers, Detectors and Associated Equipment, 2001, 456, 411-417.

16. F. Boroumand, M. Zhu, A. Dalton, J. Keddie, P. Sellin and J. Gutierrez, Appl. Phys. Lett., 2007, 91, 033509.

17. A. Intaniwet, C. A. Mills, M. Shkunov, H. Thiem, J. L. Keddie and P. J. Sellin, 
J. Appl. Phys., 2009, 106, 064513.

18. A. Intaniwet, C. A. Mills, P. J. Sellin, M. Shkunov and J. L. Keddie, ACS applied materials \& interfaces, 2010, 2, 1692-1699.

19. A. Intaniwet, J. L. Keddie, M. Shkunov and P. J. Sellin, Organic Electronics, 2011, 12, 1903-1908.

20. B. Fraboni, A. Ciavatti, F. Merlo, L. Pasquini, A. Cavallini, A. Quaranta, A. Bonfiglio and A. Fraleoni-Morgera, Adv. Mater., 2012, 24, 2289-2293.

21. L. Basiricò, A. Ciavatti, M. Sibilia, A. Fraleoni-Morgera, S. Trabattoni, A. Sassella and B. Fraboni, IEEE Trans. Nucl. Sci., 2015, 62, 1791-1797.

22. A. Ciavatti, E. Capria, A. Fraleoni-Morgera, G. Tromba, D. Dreossi, P. J. Sellin, P. Cosseddu, A. Bonfiglio and B. Fraboni, Adv. Mater., 2015, 27, 7213-7220.

23. G. Pipan, M. Bogar, A. Ciavatti, L. Basiricò, T. Cramer, B. Fraboni and A. Fraleoni-Morgera, Adv Mater Interfaces, 2018, 5, 1700925.

24. A. Ciavatti, T. Cramer, M. Carroli, L. Basiricò, R. Fuhrer, D. De Leeuw and B. Fraboni, Applied Physics Letters, 2017, 111, 183301.

25. H. Thirimanne, K. Jayawardena, A. Parnell, R. Bandara, A. Karalasingam, S. Pani, J. Huerdler, D. Lidzey, S. Tedde and A. Nisbet, Nat. Commun., 2018, 9, 2926.

26. h. h. o. h. ANSI/HPS N43.17. Radiation Safety for Personnel Security Screening Systems Using X-Ray or Gamma Radiations (HPS.

27. D. J. Brenner, C. D. Elliston, E. J. Hall and W. E. Berdon, American journal of roentgenology, 2001, 176, 289-296.

28. O. D. Jurchescu, J. Baas and T. T. M. Palstra, Applied Physics Letters, 2004, 84, 3061-3063.

29. A. Troisi, Advanced Materials, 2007, 19, 2000-2004.

30. B. Fraboni, C. Femoni, I. Mencarelli, L. Setti, R. Di Pietro, A. Cavallini and A. Fraleoni-Morgera, Adv. Mater., 2009, 21, 1835-1839.

31. T. Higashi and K. Osaki, Acta Crystallogr B, 1977, 33, 607-609.

32. G. Olivieri, A. Cossaro, E. Capria, L. Benevoli, M. Coreno, M. De Simone, K. C. Prince, G. Kladnik, D. Cvetko and B. Fraboni, The Journal of Physical Chemistry C, 2014, 119, 121-129.

33. P. Hartman and H.-K. Chan, Pharmaceutical research, 1993, 10, 1052-1058.

34. A. Malkin, Y. G. Kuznetsov, T. Land, J. DeYoreo and A. McPherson, Nature structural biology, 1995, 2, 956.

35. X. Zeng, Y. Qiu, J. Qiao, G. Dong and L. Wang, Applied surface science, 2007, 253, 3581-3585.

36. J. Yang, H.-H. Fang, R. Ding, S.-Y. Lu, Y.-L. Zhang, Q.-D. Chen and H.-B. Sun, The Journal of Physical Chemistry C, 2011, 115, 9171-9175.

37. W.-K. Burton, N. Cabrera and F. Frank, Philosophical Transactions of the Royal Society of London. Series A, Mathematical and Physical Sciences, 1951, 243, 299-358.

38. R. Boistelle and J. Astier, Journal of Crystal Growth, 1988, 90, 14-30. 
39. J. Ziegler, see http://www. srim. org, 2013.

40. A. Ciavatti, P. Sellin, L. Basiricò, A. Fraleoni-Morgera and B. Fraboni, Applied Physics Letters, 2016, 108, 153301.

41. M. Thompson, S. L. Ellison and R. Wood, Pure and Applied Chemistry, 2002, 74, 835-855.

42. Q. Ou, Y. Zhang, Z. Wang, J. A. Yuwono, R. Wang, Z. Dai, W. Li, C. Zheng, Z. Q. Xu and X. Qi, Adv. Mater., 2018, 30, 1705792.

43. Z. Li, H. Qiao, Z. Guo, X. Ren, Z. Huang, X. Qi, S. C. Dhanabalan, J. S. Ponraj, D. Zhang and J. Li, Adv. Funct. Mater., 2018, 28, 1705237.

44. Y. Liu, B. N. Shivananju, Y. Wang, Y. Zhang, W. Yu, S. Xiao, T. Sun, W. Ma, H. $\mathrm{Mu}$ and S. Lin, ACS applied materials \& interfaces, 2017, 9, 36137-36145.

45. Z. Xie, C. Xing, W. Huang, T. Fan, Z. Li, J. Zhao, Y. Xiang, Z. Guo, J. Li and Z. Yang, Adv. Funct. Mater., 2018, 28, 1705833.

46. I. Clairand, J. M. Bordy, E. Carinou, J. Daures, J. Debroas, M. Denozière, L. Donadille, M. Ginjaume, C. Itié, C. Koukorava, S. Krim, A. L. Lebacq, P. Martin, L. Struelens, M. Sans-Merce and F. Vanhavere, Radiation Measurements, 2011, 46, 1252-1257.

47. A. Fraleoni-Morgera, L. Benevoli and B. Fraboni, Journal of Crystal Growth, 2010, 312, 3466-3472.

\section{Table of Contents artwork}

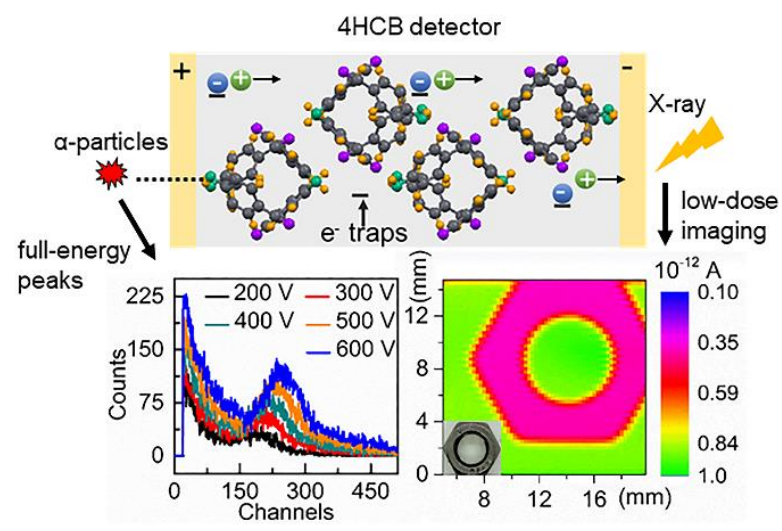

The purely organic 4HCB single crystals with high hole mobility show a capability of direct detecting ${ }^{241} \mathrm{Am} 5.49 \mathrm{MeV} \alpha$ particles and X-ray imaging under the low-dose rate (below $50 \mu \mathrm{Gyair} \mathrm{s}^{-1}$ ) exposure. 